\title{
An Easily Implementable Network Analyzer
}

\author{
KENZO WATANABE, MEMBER, IEEE, AND MITSUAKI ASHIKI
}

\begin{abstract}
A simple network analyzer is devised which makes use of the sampling and time-division multiplexing techniques to detect a signal in a phasor form. Composed of a binary $90^{\circ}$ phase shifter and a coherent detector, the detection system involved allows the real and imaginary parts of a scattering parameter to be detected sequentially. The performance of a prototype analyzer supports the detection principle. The error analysis is also given to specify the operation of a binary $90^{\circ}$ phase shifter needed for the analyzer to assure a prescribed measurement accuracy.
\end{abstract}

\section{INTRODUCTION}

$\mathbf{T}$ HE MEASUREMENT of a scattering parameter is indispensable for characterization of microwave networks and devices. A common way to measure an $s$ parameter is to use either a slotted line or a network analyzer [1]. A slotted line method provides accurate data, but is laborious and time consuming. A network analyzer, on the other hand, permits quick measurements, but the available commercial instrument is expensive.

This paper describes a microwave network analyzer implementable without any sophisticated component. The essential part is a binary $90^{\circ}$ phase shifter in combination with a coherent detector. This combination makes possible the time-sharing detection of the real and imaginary parts of an $s$-parameter to be measured.

Manuscript received May 2, 1977.

The authors are with the Research Institute of Electronics, Shizuoka University, Johoku 3-5-1, Hamamatsu 432, Japan.
Following this introductory section, Section II develops the time-domain analysis which describes the time-sharing detection. The analysis assumes the binary $90^{\circ}$ phase shifter involved to be ideal. A performance of the real phase shifter differs, inevitably, from that of the ideal one. Therefore, the measurement error is evaluated in Section III in terms of the mismatch factors of the binary $90^{\circ}$ phase shifter. Section IV describes the performance of a prototype analyzer together with a measuring example which confirms the detection principle.

\section{PRinCiPles of OpERATION}

Fig. 1 shows a configuration of the network analyzer. It consists of three main sections-a sweep generator, microwave bridge, and video signal processor. The sweep generator is assumed to be already available and to provide the leveled RF power over the whole frequency range of measurement.

The microwave bridge is composed of the reference and test channels each decoupled from the other by means of $3-\mathrm{dB}$ couplers. An adjustable line stretcher is incorporated into the reference channel to equalize the apparent electrical lengths of the two channels. The wave in the test channel is applied to the component under test and is subjected to the amplitude and phase variations in accordance with the magnitude and the phase of the $s$-parameter, respectively. This signal is routed to a binary $90^{\circ}$ 


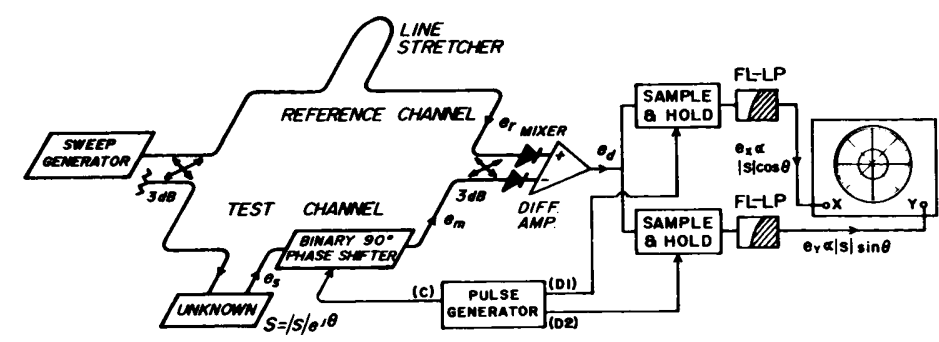

Fig. 1. A schematic diagram of a microwave network analyzer.

phase shifter driven by the modulating pulse generator, where it undergoes the phase shift of $90^{\circ}$ during a halfperiod of modulation frequency, and no phase shift, apart from the propagation delay, during another half period. This phase-shift keyed signal is mixed with a reference carrier in mixer diodes to be converted to a video signal.

The video signal thus obtained consists of an alternate sequence of two components, one proportional to the real part and the other to the imaginary part of the $s$-parameter, as described shortly. The two components are separated from the time sequence to each individual component by the sample-and-holds in synchronism with the binary $90^{\circ}$ phase shifter.

The time-domain analysis makes the time-sharing detection process clear. Assuming that the wave incident upon the unknown has a unit amplitude, the signal $e_{s}(t)$ is written as

$$
e_{s}(t)=\operatorname{Re}\{S \cdot \exp (j \omega t)\}=S_{r}(t) \cos \omega t-S_{i}(t) \sin \omega t
$$

where $S=|S| \exp (j \theta)=S_{r}(t)+j S_{i}(t)$ is the $s$-parameter being measured. The transfer function $H(f)$ of the binary $90^{\circ}$ phase shifter is given by

$$
\begin{aligned}
& H(f) \\
& =\left\{\begin{array}{l}
1, \quad(n-1 / 4) T_{m} \leq t \leq(n+1 / 4) T_{m} \\
-j \operatorname{sgn} f, \quad(n+1 / 4) T_{m} \leq t \leq(n+3 / 4) T_{m}
\end{array}\right.
\end{aligned}
$$

where $T_{m}$ is the modulation period and $n$ is an integer. The phase-shift-keyed signal is thus given by

$$
\begin{aligned}
e_{m}(t)=\sum_{n=-\infty}^{\infty}\left(e_{s}(t) \Pi(\right. & \left.t-n T_{m}\right) \\
& \left.+\hat{e}_{s}(t) \Pi\left(t-(2 n+1) T_{m} / 2\right)\right)
\end{aligned}
$$

where $\hat{e}_{s}(t)$ is the Hilbert transform of $e_{s}(t)$, and $\Pi(t-$ $n T_{m}$ ) denotes a switching function that assumes 1 during the time interval $T_{m} / 2$ centered at $t=n T_{m}$, and 0 elsewhere [2].

The mixer operates as a product demodulator. Besides a product component, it produces a dc component contributed independently by the signal and the reference carrier, but it is canceled out by taking the difference between the demodulated outputs of the two mixers. Assuming that $T_{m}$ is short enough so that the $s$-parameter does not change appreciably during $T_{m}$, which places the lower limit on the modulation frequency, we have the demodulated output

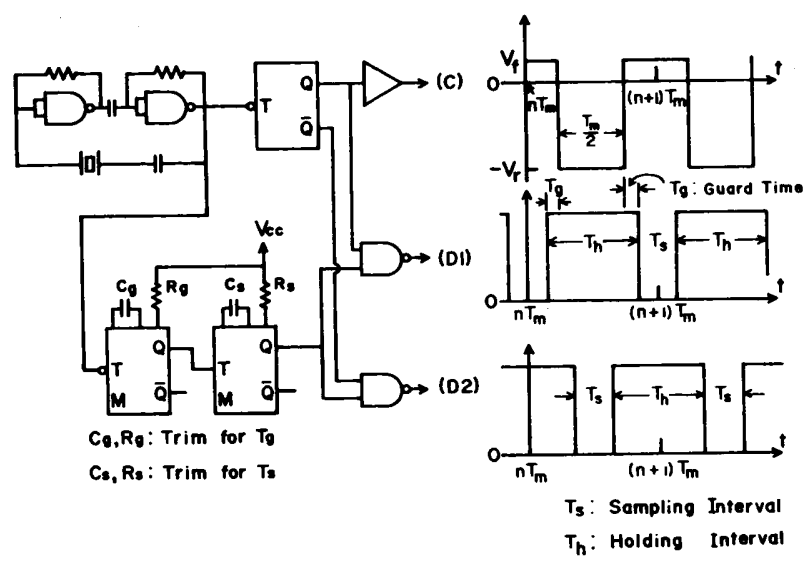

Fig. 2. An implementation of the pulse generator and the generated pulse patterns.

$$
\begin{aligned}
& e_{d}(t)=e_{m}(t) \cos \omega t \\
& =\sum_{n=-\infty}^{\infty}\left(S_{r}\left(n T_{m}\right) \Pi\left(t-n T_{m}\right)\right. \\
& \left.\quad+S_{i}\left(n T_{m}\right) \Pi\left(t-(2 n+1) T_{m} / 2\right)\right)
\end{aligned}
$$

where the double-frequency term is deleted because of a low-pass filter in the video portion of the mixer. The demodulated output is thus the time-division multiplexed version of the real and imaginary parts of the $s$-parameter. It is understood from this that the binary $90^{\circ}$ phase shifter plays, in combination with the coherent detector, a sampler and commutator part in a time-division multiplexing system.

The sample-and-hold plays a decommutator part in a demultiplexer. The timing between the commutator and the decommutator is shown in Fig. 2 together with an implementation of a pulse generator. Fig. 2 supposes the binary $90^{\circ}$ phase shifter employing a $\mathrm{p}$-i-n diode which causes no phase-shift when forward-biased by $V_{f}$ and a $90^{\circ}$ phase-shift when reverse-biased by $V_{r}$. The guard time is also provided to avoid the crosstalk between the signals in neighboring time slots which would otherwise be caused by the finite bandwidth of the differential amplifier [3] and the finite aperture and acquisition times of the sampleand-hold. Referring to (4) and Fig. 2, it is recognized that the output of the sample-and-hold driven by the pulse train $(D 1)$ is a step approximation to $S_{r}(t)$, with each step having the width $T_{m}$. Similarly, that by the pulse train $(D 2)$ is a step approximation to $S_{i}(t)$. The smoothed outputs are provided by additional low-pass filters (FL-LP's in Fig. 1).

\section{MEASUREMENT ERROR}

The allowable tolerance for a measurement error determines the available bandwidth of a network analyzer. In the present scheme, the most serious error source is the mismatch of the binary $90^{\circ}$ phase shifter. With the mismatch present, the transfer function $(2 \mathrm{~b})$ has to be modified to

$$
H(f)=(1-\epsilon) \exp \{-j(\pi / 2 \pm \delta) \operatorname{sgn} f\}
$$




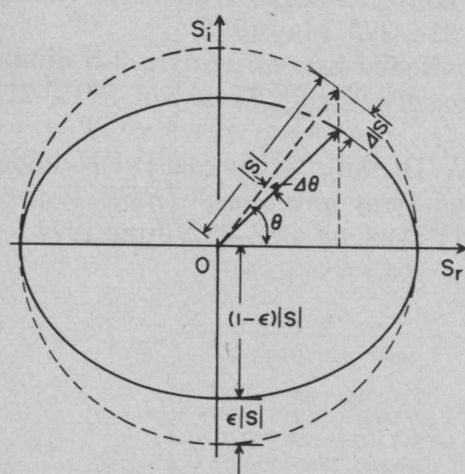

(a)

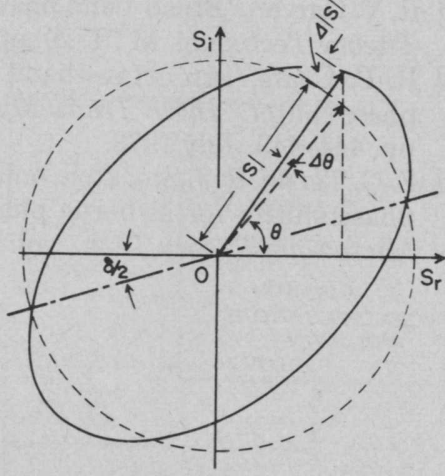

(b)
Fig. 3. The erroneous indications (solid vectors) of an $s$-parameter due to the amplitude unbalance and the phase-shift deviation of the binary $90^{\circ}$ phase shifter. The dotted vectors show the corresponding true values. The ellipses and the dotted circles are the loci of the erroneous and corresponding true $s$-parameters when $\theta$ is varied over $2 \pi$ rad. (a) The amplitude unbalance. (b) The phase-shift deviation.

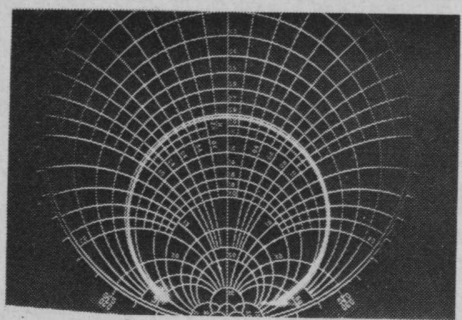

(a)

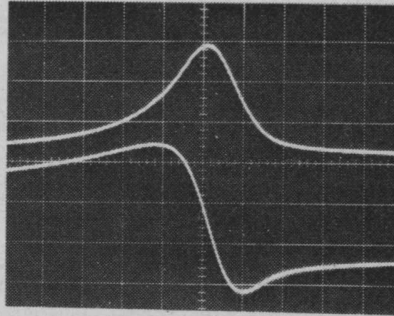

(b)
Fig. 4. An example of measurement; an input reflection coefficient of a parametric amplifier is measured at $9.45 \mathrm{GHz}$ while sweeping the varactor bias voltage from $-6 \mathrm{~V}$ to $0.9 \mathrm{~V}$ at repetition frequency of 350 Hz. (a) Smith chart representation. (b) Two channel display of the real (upper trace) and imaginary (lower trace) parts.

where $\epsilon$ and $\delta$ represent the amplitude unbalance and the phase-shift deviation, respectively, characterizing the mismatch. Their contributions to the measurement error can be evaluated separately.

The amplitude unbalance reduces the demodulated imaginary component of the $s$-parameter by $\epsilon$. Therefore, the $s$-parameter indicated on a complex plane traces not a circle but an ellipse when $\theta$ is varied over $2 \pi \mathrm{rad}$, as shown in Fig. 3(a). The erroneous indication (solid vector) is related to the corresponding true value (dotted vector) in that both should have an identical real part for any $\theta$. One can use this relation to obtain the fractional amplitude and phase errors, with the results,

$$
\begin{gathered}
\frac{\Delta|S|}{|S|}=\frac{\epsilon(1-\cos 2 \theta)}{2} \leq \epsilon \\
\Delta \theta=\theta-\tan ^{-1}(1-\epsilon) \tan \theta \leq \epsilon /(2-\epsilon) .
\end{gathered}
$$

The amplitude error less than 5 percent requires $\epsilon$ to be less than 0.05 or $0.45 \mathrm{~dB}$. The phase error is then within $1.5^{\circ}$.

The phase-shift deviation causes the cross-talk so that the output $e_{Y}$ contains not only the imaginary component but also the real component of the $s$-parameter. The trajectory on a complex plane then becomes an ellipse whose major axis makes an angle $45^{\circ}$ with respect to the real axis, as shown in Fig. 3(b). The same relation as in Fig. 3(a) also holds between the erroneous and corresponding true indications. Using this relation, we obtain

$$
\begin{aligned}
& \frac{\Delta|S|}{|S|}=\frac{1}{2} \sin \delta \cdot \sin (\delta+2 \theta) \leq \frac{\delta}{2} \\
& \Delta \theta=\tan ^{-1}(\delta+\tan \theta)-\theta \leq \delta .
\end{aligned}
$$

When $\delta$ is less than $5^{\circ}$ or $0.09 \mathrm{rad}$, the amplitude and phase errors are within 5 percent and $5^{\circ}$, respectively.

\section{PRototype ANALyzer}

A prototype network analyzer has been implemented using $X$-band waveguide components. The binary $90^{\circ}$ phase shifter consists of a circulator and a high speed $p-i-n$ diode mounted $\lambda_{g} / 8$ away from a short. The specified frequency is $9.5 \mathrm{GHz}$. Taking the slew rate of the differential amplifier and the response speed of the sampleand-hold into account, the modulation frequency of 200 $\mathrm{kHz}$ is chosen. This will suffice for most measurements. The guard time is $0.5 \mu \mathrm{s}$.

Fig. 4(a) is an example of measurement, showing a reflection coefficient locus of a parametric amplifier. The measurement is made at $9.45 \mathrm{GHz}$ while sweeping the bias voltage of a varactor diode. Fig. $4(\mathrm{~b})$ is the two channel display of the same reflection coefficient, demonstrating the separate detection of the real and imaginary components.

Since the emphasis is placed on the experimental confirmation of the detection principle, no special attention is paid for broad-banding the binary $90^{\circ}$ phase shifter; consequently, the phase-shift deviation increases monotonically as the frequency of measurement deviates from the specified one to reach $\pm 5^{\circ}$ at $9500 \pm 300 \mathrm{MHz}$. The available bandwidth over which the amplitude and phase errors stay within 5 percent and $5^{\circ}$, respectively, is therefore only about 6 percent. For the same error tolerance, however, the available bandwidth over 40 percent is quite possible by using any one of those broad-band $90^{\circ}$ phase shifter reported by Schiffman [4], Garver [5], Coats [6], and Terrio et al. [7].

\section{CONCLUSIONS}

A novel scheme for implementing a microwave network analyzer has been presented. It features easy implementation made possible by the time-divided phasor detection of a signal. The principles of detection has been experimentally confirmed by the performance of the prototype analyzer.

The available bandwidth of the prototype analyzer is rather narrow because of the poor performance of the binary $90^{\circ}$ phase shifter involved. A broad-band $90^{\circ}$ diode phase shifter is now in design to extend the available bandwidth.

\section{REFERENCES}

[1] B. M. Oliver and J. M. Cage, Electronic Measurements and Instrumentation. New York: McGraw-Hill, 1971, pp. 654-703. 
[2] A. B. Carlson, Communication Systems. New York: McGraw-Hill, 1968, pp. 31-33, 104-110.

[3] P. F. Panter, Modulation, Noise, and Spectral Analysis. New York: McGraw-Hill, 1965, pp. 557-565.

[4] B. M. Schiffman, "A new class of broad-band microwave 90-degree phase shifters," IRE Trans. Microwave Theory Tech., vol. MTT-6, pp. 232-237, Apr. 1958.
[5] R. V. Garver, "Broad-band phase shifters," IEEE Trans. Microwave Theory Tech., vol. MTT-20, pp. 314-323, May 1972.

[6] R. P. Coats, "An octave-band switched-line microstrip 3-b diode phase shifter," IEEE Trans. Microwave Theory Tech., vol. MTT-21, pp. 444-449, July 1973.

[7] F. G. Terrio, R. J. Stockton, and W. D. Sato, "A low cost P-I-N diode phase shifter for airborne phased-array antennas," IEEE Trans. Microwave Theory Tech., vol. MTT-22, pp. 688-692, June 1974. 